\title{
The extent of small fibre sensory neuropathy in diabetics with plantar foot ulceration
}

\author{
Z ALI, M CARROLL, K P ROBERTSON, CLARE J FOWLER \\ From the Reta Lila Weston Institute of Neurological Studies, The Middlesex Hospital Medical School, and The \\ London Foot Hospital, London, UK
}

SUMMARY Thresholds for cutaneous warming and cooling stimuli were measured in 20 diabetics with neuropathic foot ulcers. All patients had a profound disturbance of sensory perception in the ulcerated foot with complete loss of perception of warming; thresholds for vibration and cooling were highly abnormal in all but two patients. Measurements of thermal threshold were made on both feet in 10 patients: warming was lost bilaterally in all, and cooling was bilaterally absent in six. There was no clear pattern of sensory loss in those diabetics with unilateral foot ulceration to suggest that sensory impairment was the determining factor for the development of a plantar ulcer. Measurements of thermal thresholds were made at additional sites in 13 patients and although the most marked abnormalities of sensation were always found in the feet, in some severe neuropaths, abnormal thresholds on the hand and even the face were demonstrated. Thresholds for warming were invariably more abnormal than thresholds for cooling. The diabetics with neuropathic ulceration in this study all had severe generalised peripheral nerve disease involving large myelinated as well as both small myelinated and unmyelinated sensory fibres. The quantitative evidence on the distribution of sensory loss for thermal sensations supports the hypothesis that the neuropathic process affecting the small myelinated and unmyelinated fibres is length dependent.

Although loss of sensation is not the sole aetiological factor in the pathogenesis of diabetic plantar ulceration,' profound neuropathy does appear to be an essential feature for the development of this complication. The view that ulceration occurs as a consequence of repeated mechanical trauma on an insensitive $\operatorname{sole}^{23}$ is probably an over simplification and the loss of other neural functions, such as those subserved by the autonomic innervation may alse be important.

It has long been recognised that painless plantar foot ulcers occur in diabetics with severe generalised neuropathy. More recently, emphasis has been placed on the severity of the small myelinated and unmyelinated fibre dysfunction that occurs in these patients. The occurrence of a generalised autonomic neuropathy was demonstrated by studies of cardiovascular reflexes which showed particularly severe defects in diabetics with foot ulcers. ${ }^{4-6}$ Means of specifically

Address for reprint requests: Dr Clare J Fowler, Department of Clinical Neurophysiology, The Middlesex Hospital, London WIN 8AA, UK.

Received 24 June 1988.

Accepted 26 August 1988 studying autonomic function in the lower limb have now been devised; the volume of sweat produced by a small area of skin on the dorsum of the foot following stimulation of the sympathetically mediated axon reflex by local electrophoresis of acetlycholine, has been measured. ${ }^{7}$ A reduction in sweat volume was found in diabetics, and this was particularly marked in patients with foot ulcers. ${ }^{8}$ The C-fibre nociceptive flare response so evoked has now been quantified ${ }^{9}$ and severe abnormality has been shown in diabetics with foot ulceration indicating impairment of nociceptive $C$ fibre function. ${ }^{10}$ Both the loss of physiological sweating and the protective role of pain sensation as well as a loss of the normal inflammatory response may predispose to ulcer formation.

Other measures of small fibre dysfunction in diabetics with neuropathic foot lesions have also been carried out. Le Quesne and Fowler ${ }^{11}$ measured pain thresholds to pinch on the dorsum of the foot in 16 feet affected by plantar ulceration but were not able to demonstrate an invariable abnormality in this group. Using a thermal testing system which measures hotcold limen Guy and his colleagues ${ }^{12}$ examined 10 patients with foot ulcers, amongst a larger group of 


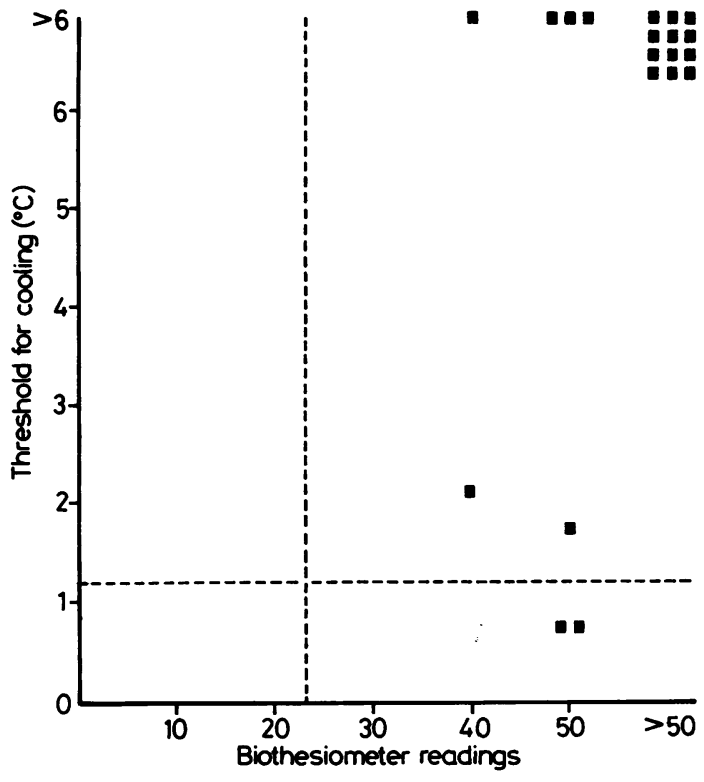

Fig 1 Threshold for perception of cooling against threshold for vibration (Biothesiometer units). Readings shown are from ulcerated feet. The dotted lines indicate the upper limits of normal found in control male subjects aged under 65 years.

diabetics. They found a severe deficit in all patients with ulcers and found temperature appreciation subserved by $A$ delta and $C$ fibres more frequently abnormal than vibration perception, a sensation conveyed by $A$ beta fibres.

We have used a temperature testing system that measures thresholds for warming and cooling separately, ${ }^{13}$ thus assessing the integrity of the sensory unmyelinated and small myelinated fibres respectively. ${ }^{14}$ With this we quantified the extent and severity of sensory loss in patients with diabetic plantar ulceration.

\section{Patients}

Thermal thresholds were measured in 20 diabetics who were attending the London Foot Hospital for treatment of plantar ulceration. There were three females and 17 males, with an age range of between 32 and 65 years, mean 50.3 years. The patients were assessed by one of us (KR) and were considered to have neuropathic ulceration occurring on the weight bearing aspect of either the big toe, the metatarsal heads, the midfoot or on the heel, due to repeated trauma. It was also observed that deep debridment of ulcers was a painless procedure to these patients. Eleven patients had either a past history of bilateral plantar ulcers or active ulcers on both feet at the time they were seen.
Methods

Measurements of cutaneous thermal thresholds were made using a portable thermal threshold testing system. ${ }^{13}$ Thresholds for vibration on the halluces were measured using a Biothesiometer (Biomedical Instrument Co., Newbury, Ohio).

In all 20 patients, thresholds on the sole of the foot with the past or present ulceration were measured. In 10 patients, five of whom had bilateral ulceration, the other foot was also tested. In 13 patients measurements of thresholds were made on the dorsum of the hand in an area of skin innervated by the superficial radial nerve and on the face just lateral to the nose.

\section{Results}

Results are expressed here either as the absolute figure for threshold in ${ }^{\circ} \mathrm{C}$, that is, observed value or, because of the considerable age and sex variability of thermal thresholds, as "abnormality" in ${ }^{\circ} \mathrm{C}$. To obtain a figure for "abnormality" the expected value for the patient's age and sex, derived from 2 standard deviations of a regression line formerly obtained from control data, ${ }^{13}$ was subtracted from the observed value.

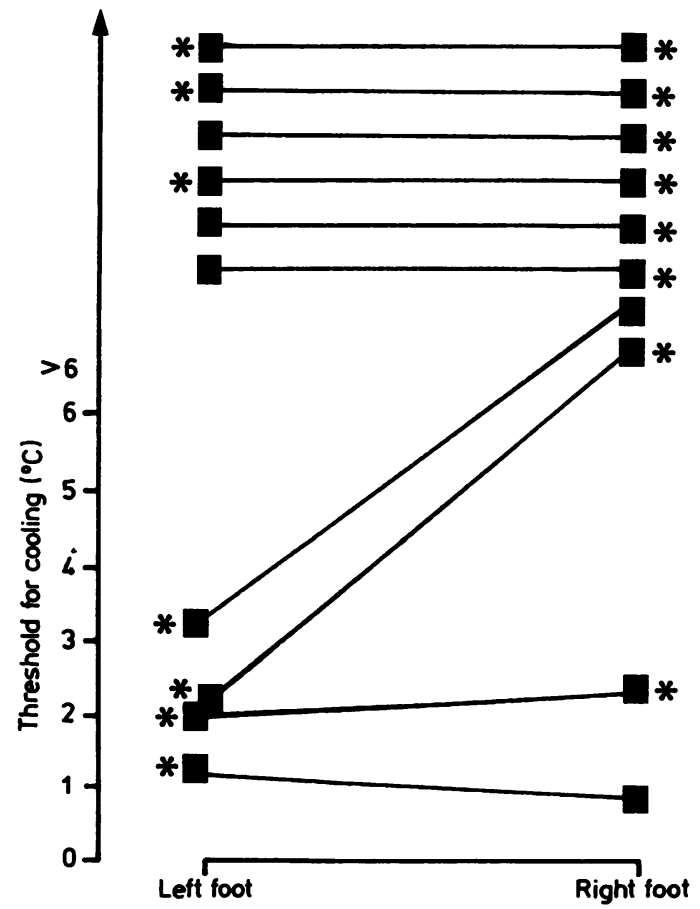

Fig 2 The differences in cooling thresholds between the two feet of 10 patients with neuropathic plantar foot ulcers. (*Ulcerated feet) 


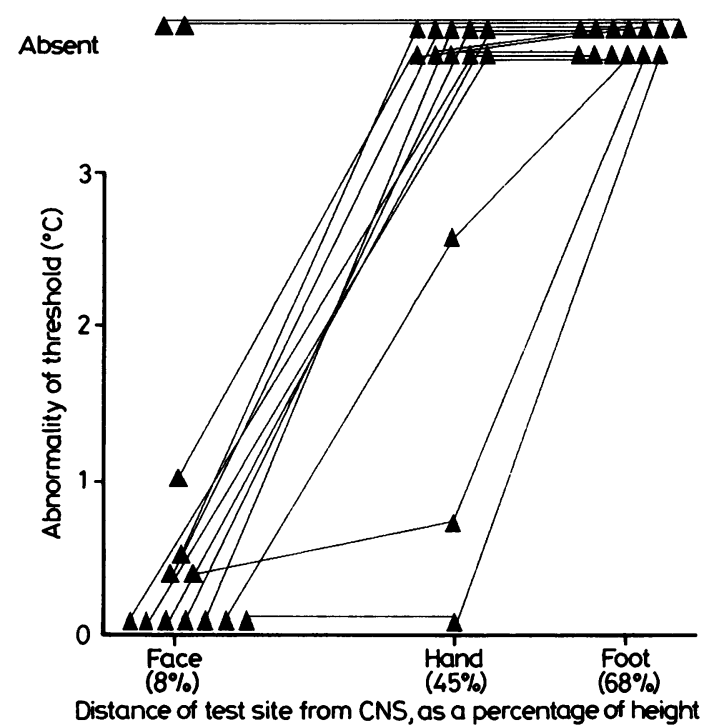

Figure 3 The abnormalities of warming thresholds at the three different sites tested. The figures on the $x$ axis refer to the estimated length of peripheral nerve innervating each site expressed as a percentage of height.

The measuring system employed measures up to $6^{\circ} \mathrm{C}$ change for warming and cooling. The upper limit of threshold for those aged under 65 years on the sole of the foot in men and women respectively is 3.5 and $2 \cdot 4^{\circ} \mathrm{C}$ for warming and 1.2 and $0.8^{\circ} \mathrm{C}$ for cooling.

Results from this study show four main points.

1. Extent of impairment of three sensory modalities tested.

In all 20 patients there was a complete loss of perception of warming and a severe abnormality of vibration perception. Perception of cooling was within normal limits in two patients, abnormally raised in two and otherwise lost (fig 1).

2. Extent of neuropathy in ulcerated and non-ulcerated feet.

In the 10 patients in whom measurements were made on the soles of both feet, five had either a past history of bilateral ulceration or had active ulcers affecting both feet. All 10 diabetics had lost perception of warming bilaterally. Cooling perception was lost in both feet in six patients but measurable at least unilaterally in the remaining four (fig 2). Overall no clear pattern was shown which might have indicated relative sparing of perception of cooling in the nonulcerated foot.

3. Evidence that small fibre neuropathy may be length dependent.

To examine the possibility that diabetic small fibre neuropathy may be length dependent, data were derived using surface measurements to estimate the approximate lengths of the peripheral nerves innervating the test sites, length being given as a percentage of the subjects' height. Figs 3 and 4 show abnormalities in thermal perception, adjusted for age and sex for threshold at each site.

4. Differences in abnormality of perception for cooling and warming.

The results from the 10 patients tested at three different sites show the perception of warming is more abnormal than that for cooling. This is particularly evident in three subjects with highly abnormal warming thresholds at one or more sites and comparatively normal thresholds for cooling (fig 5).

\section{Discussion}

Characteristically neuropathic plantar ulcers in diabetics are painless, with profound sensory loss on the sole of the foot being a critical aetiological factor in the pathogenesis of such a lesion. Although mechanical, vascular and connective tissue changes have a causative role in the development of diabetic foot ulcers, in clinical practice these lesions are usually found only in patients with severe generalised peripheral neuropathy.

Boulton et al ${ }^{15}$ showed that the Biothesiometer, used to measure vibration perception threshold in the great toes in diabetics with foot ulceration "was the most discriminative of the conventional investigations"; abnormalities of this measurement being associated with foot ulceration and the presence of abnormally high, localised pressure areas under the

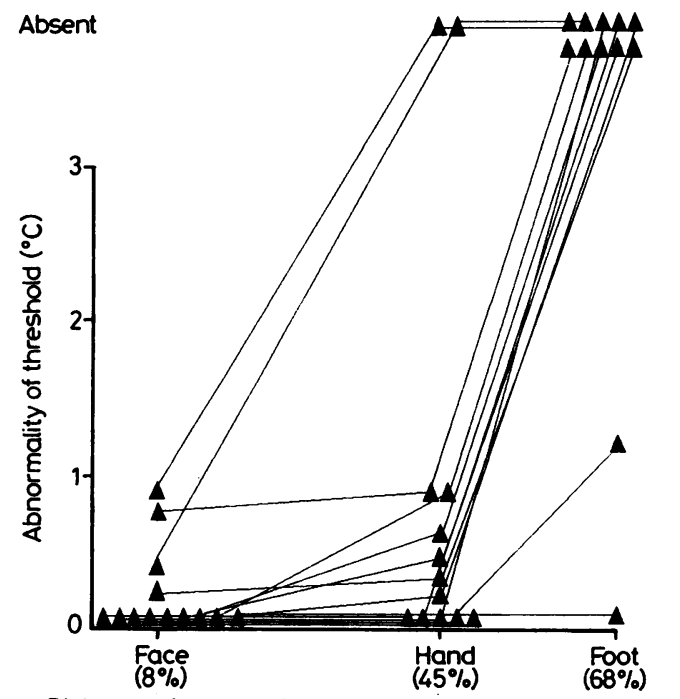

Distance of test site from CNS, as a percentage of height

Fig 4 As in fig 3 but for cooling. 


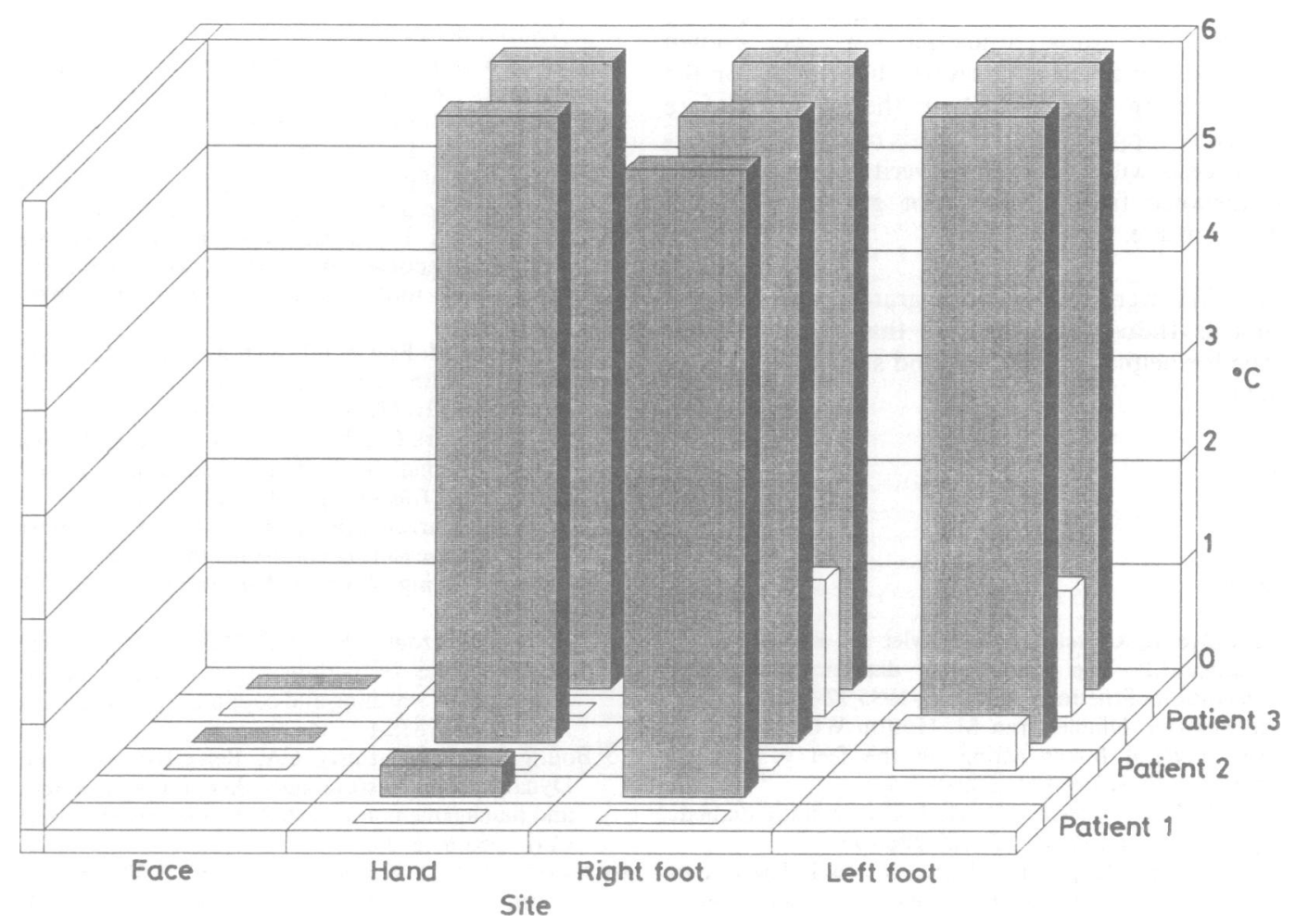

Fig 5 Three patients with abnormal warming thresholds (shaded) at one or more sites with comparatively little or no abnormality in the cooling thresholds (unshaded).

foot. Our study confirms that all patients with neuropathic ulceration have highly abnormal vibration perception thresholds indicating a severe abnormality of large myelinated fibre dysfunction. Guy et $a l^{12}$ did not separate out those with plantar ulceration from other diabetics when presenting their results showing impairment of thermal perception to be more abnormal than vibration perception.

Not unexpectedly this study has demonstrated severe sensory impairment in the feet with complete loss of perception of warming bilaterally. Perception of warming is the earliest indicator of unmyelinated fibre dysfunction. ${ }^{12}$ Loss of perception of warming was shown to be present in a group of men with diabetic impotence; ${ }^{16}$ a complication of diabetes that may occur so early as a feature of diabetic autonomic neuropathy that cardio-vascular reflexes are still normal. The fact that perception of cooling was relatively preserved in a small proportion of cases may merely reflect the fact that this is a less subtle psychophysical task but the observation is also consistent with the hypothesis that unmyelinated fibres conveying the sensation of warming are more severely affected than small myelinated fibres which convey sensations of cooling. ${ }^{14}$

In just over half the patients in this study, plantar ulceration was bilateral and it is perhaps surprising that the problem is not more often symmetrical when complicating a generalised neuropathy. It is possible that structural deformity of the foot, unrelated to diabetes, would cause a unilateral site that is subjected to increased mechanical trauma. No studies have shown a significantly worse impairment of perception of pain, sweating or flare response on the side of ulcer formation in the unilateral condition and we found no systematic difference between ulcerated and nonulcerated feet for the perception of cooling.

A proximo-distal gradient of sensory loss in diabetic neuropathy has been recognised ${ }^{17}$ and Said et al ${ }^{18}$ described centripetal degeneration of axons in small fibre diabetic polyneuropathy. Consideration of the remarkable spatial geometry of an unmyelinated fibre innervating the foot, that is, $1 \mu$ in diameter, $1000000 \mu$ in length, makes this an attractive hypothesis. Although the values for threshold at different anatomical sites vary, we expressed the values 
obtained on the face, hand and foot in terms of "abnormality", thus adjusting for the known variability. Our findings of severe abnormality on the foot, becoming less marked on the hand and face respectively support the hypothesis of a length dependent process, with increasing severity of abnormality with distance from dorsal root ganglion to skin innervation site.

$\mathrm{ZA}$ and $\mathrm{MC}$ were supported by grants from Imperial Chemicals Industries PLC. We thank Dr P M Le Quesne for helpful discussions and suggestions about the text.

\section{References}

1 Delbridge L, Ctercteko G, Fowler C, Reeve TS, Le Quesne LP. The aetiology of diabetic neuropathic ulceration of the foot. Br J Surg 1985;72:1-6.

2 Ctercteko G, Dhanendran M, Hutton WC, Le Quesne LP. Vertical forces acting on the feet of diabetic patients. Br J Surg 1981;68:608-14.

3 Pollard JP, Le Quesne LP. Method of healing diabetic forefoot ulcers. $\mathrm{Br}$ Med $J$ 1983;286:436-7.

4 Deanfield JE, Daggett PR, Harrison MJG. The role of autonomic neuropathy in diabetic foot ulceration. $J$ Neurol Sci 1980;47:203-10.

5 Edmonds ME, Nicolaides $K$, Watkins $P$. The importance of autonomic neuropathy in the aetiology of the diabetic neuropathic foot. Diabetologia 1981;21:506-7.

6 Ahmed ME, Delbridge L, Le Quesne LP. The role of autonomic neuropathy in diabetic foot ulceration. $J$ Neurol Neurosurg Psychiatry 1986;49:1002-6.

7 Low PA, Caskey PE, Tuck RR, Fealey RD, Dyck PJ.
Quantitative sudomotor axon reflex test (QSART). Ann Neurol 1983;14:573-80.

8 Ahmed ME, Le Quesne PM. Quantitative sweat test in diabetics with neuropathic foot lesions. J Neurol Neurosurg Psychiatry, 1986;49:1059-62.

9 Parkhouse N, Le Quesne PM. Quantitative objective assessment of peripheral nociceptive $\mathrm{C}$ fibre function. I Neurol Neurosurg Psychiatry 1988;51:28-34.

10 Parkhouse N, Le Quesne PM. Impaired neurogenic vascular response in patients with diabetes and neuropathic foot lesions. $N$ Eng $J$ Med 1988;318: 1306-9.

11 Le Quesne PM. Fowler CJ. A study of pain thresholds in diabetics with neuropathic foot lesions. J Neurol Neurosurg Psychiatry 1986;49:1191-4.

12 Guy RJC, Clark CA, Malcolm PN, Watkins PJ. Evaluation of thermal and vibration sensation in diabetic neuropathy. Diabetologia 1988;28:131-7.

13 Fowler CJ, Carroll MB, Burns D, Howe N. A portable system for measuring cutaneous thresholds for warming and cooling. J Neurol Neurosurg Psychiatry 1987; 50:1211-5.

14 Fowler CJ, Sitzoglou K, Ali Z, Halonen P. The conduction velocities of peripheral nerve fibres conveying sensations of warming and cooling. J Neurol Neurosurg Psychiatry 1988;51:1164-70.

15 Boulton AJM, Hardisty CA, Betts RP, et al. (1983) Dynamic foot pressure and other studies as diagnostic and management aids in diabetic neuropathy. Diabetes Care 1983;6:26-33.

16 Fowler CJ, Ali Z, Kirby RS, Pryor JP. (1988) The value of testing for unmyelinated fibre sensory neuropathy in diabetic impotence. Br J Urol 1988;61:63-7.

17 Sabin TD, Geschwind N, Waxman SG. Patterns of clinical deficits in peripheral nerve disease. In: Waxman SG, ed Physiology and Pathobiology of Axons. New York, Raven Press. 1978.

18 Said G, Slama G, Selva J. Progressive centripetal degeneration of axons in small fibre diabetic polyneuropathy. Brain 1983;106:791-807. 